\title{
An in-frame complex germline mutation in the juxtamembrane intracellular domain causing RET activation in familial medullary thyroid carcinoma
}

\author{
Daniela Cordella ${ }^{1 *}$, Marina Muzza ${ }^{2 *}$, Luisella Alberti ${ }^{1}$, Paolo Colombo ${ }^{3}$, \\ Pietro Travaglini ${ }^{2,3}$, Paolo Beck-Peccoz ${ }^{2,4}$, Laura Fugazzola ${ }^{2,4}$ \\ and Luca Persani ${ }^{1,2}$
}

${ }^{1}$ Istituto Auxologico Italiano, 20095 Cusano, Milan, Italy

${ }^{2}$ Department of Internal Medicine, University of Milan, 20122 Milan, Italy

${ }^{3}$ Department of Endocrinology, Istituto Clinico Humanitas IRCCS, 20089 Rozzano, Milan, Italy

${ }^{4}$ Fondazione Policlinico, IRCCS, 20122 Milan, Italy

(Requests for offprints should be addressed to L Fugazzola; Email: I.fugazzola@ policlinico.mi.it)

*(D Cordella and M Muzza contributed equally to this work)

\begin{abstract}
Activating mutations of the RET proto-oncogene are associated with inherited syndromes, multiple endocrine neoplasia (MEN2A/2B) and with familial and sporadic medullary thyroid cancer (MTC). Single base pair missense mutations in the extracellular Cys-rich domain are responsible for most MEN2A and familial MTC (FMTC) cases. Rarely, somatic deletions and germline duplications have been described in sporadic MTC and in FMTC. We report the detection and functional studies of a deletion/insertion in exon 11 (c.2646delGinsTTCT) associated with FMTC. This in-frame complex rearrangement leads to an Asn to Lys change (Lys666Asn) and to a Ser insertion. The mutation was found in the proband, who was diagnosed with metastatic MTC at 41 years, and in her son, who presented diffuse C-cells hyperplasia at 4 years of age. The mutation displayed a transforming activity stronger than Ret wild type (Ret-WT) at the focus formation assay and functional analyses after transient and stable transfection revealed an increased autophosphorylation, indicating the constitutive activation of the receptor. The transforming activity may be favoured by an increased stabilization of the fully mature form of the mutant receptor. Dimerization assay demonstrated that the activation mechanism of the complex mutation is not mediated by stable dimer formation. Computational analysis predicted nonconservative alterations in the mutant protein consistent with a possible modification of the conformation of the receptor. In conclusion, the first molecular studies on a complex germline RET mutation lying in the juxtamembrane region of the receptor are reported. Functional analyses showed that alterations at this level too can lead to a ligand independent Ret activation.
\end{abstract}

Endocrine-Related Cancer (2006) 13 945-953

\section{Introduction}

The RET proto-oncogene on human chromosome 10q11.2 (OMIM\#164771) encodes a membrane tyrosine kinase receptor, which is expressed in neural crestderived cells. It comprises extracellular, transmembrane and cytoplasmic domains. The extracellular sequences include regions with homology to the cadherin family and a large Cys-rich region. The intracellular tyrosine kinase domain is similar to that of the other receptor tyrosine kinases and signals by auto-phosphorylation of key tyrosine residues that recruit and activate $\mathrm{SH} 2$ ( $\mathrm{src}$ homology 2)- and phosphotyrosine binding-containing protein activating different signalling pathways (Alberti et al. 1998, Hansford \& Mulligan 2000). 
Mutations of the RET proto-oncogene have been associated with several inherited and non-inherited diseases. Inactivating mutations cause familial and sporadic Hirschsprung disease (OMIM*142623), a syndrome of congenital absence of enteric innervation (Romeo et al. 1994). Germline activating mutations have been identified as the underlying cause of multiple endocrine neoplasia type-2 (MEN2) and familial medullary thyroid carcinoma (FMTC), whereas somatic RET mutations are detectable in about $50 \%$ of sporadic MTCs (Eng \& Mulligan 1997, Hansford \& Mulligan 2000). Single-base pair changes in one of the six extracellular conserved Cys residues of the receptor occur in most cases of MEN2A and FMTC (OMIM*171400). MEN2B (OMIM*162300) and sporadic MTC are due in 95 and $80 \%$ of cases to a single missense mutation that involves one of the conserved amino acid residues in the intracellular tyrosine kinase domain of RET. In FMTC and sporadic MTC, mutations in exons 5, 8, 12, 13, 14 and 15 have also been reported. In all cases, the result is a 'gain of function' of Ret that is believed to be an early event leading to the neoplastic transformation.

Mutations other than single-base pair missense changes have rarely been described. Deletions of variable regions of the gene, from 3 bp to $20 \mathrm{~Kb}$, have been found in sporadic MTC (Kimura et al. 1995, Hofstra et al. 1996, Alemi et al. 1997, Ceccherini et al. 1997, Kalinin \& Frilling 1998, Marsh et al. 1998, Uchino et al. 1999, Quadro et al. 2001), while three different duplications ( 9 and $12 \mathrm{bp}$ ) and a $1 \mathrm{bp}$ deletion/ $13 \mathrm{bp}$ insertion, have been reported in MEN2A and FMTC families (Hoppner \& Ritter 1997, Hoppner et al. 1998, Pigny et al. 1999, Ahmed et al. 2005). Interestingly, all these variants are in frame, frequently involving deletion or insertions of Cys residues.

Very recently, a complex mutation including a $G$ deletion and a TTCT insertion at codon 666 has been reported in a Belgian FMTC family (Vandenbosch et al. 2005). In particular, the proband was a 12-year-old boy presenting with MTC with nodal metastasis (Fig. 1A). The mother and the grandfather harboured the same mutation, in the absence of specific MTC signs and/or symptoms. C-cells hyperplasia and an MTC focus of $15 \mathrm{~mm}$ was found in the mother, who was submitted to prophylactic total thyroidectomy. The functional activity of that mutation was not tested and the authors concluded that this gene variant is associated with a mild phenotype. They also hypothesized that the more severe behaviour found in the proband could be due to the association with a RET polymorphism (G691S) inherited from the father. Moreover, three other apparently unrelated families, originating from the same geographical region and harbouring the same RET mutation, have been reported in a recent meeting (Bex et al. 2006).

In the present paper, we report the functional analysis of this complex mutation found in another, apparently unrelated, family of Belgian origin.

\section{Materials and methods}

\section{Patients}

The proband is a 41-year-old Caucasian woman originating from Belgium. She was investigated for a thyroid nodule of about $1 \mathrm{~cm}$ at ultrasound. Biochemical investigations revealed a normal thyroid function with negative anti-thyroid antibodies and elevated basal calcitonin (CT) levels $(225 \mathrm{pg} / \mathrm{ml}$, normal value $<10)$. A stimulating test was positive with high CT levels $(528 \mathrm{pg} / \mathrm{ml})$ at $2 \mathrm{~min}$ after i.v. injection of pentagastrin (Peptavlon injection 3P; Cambridge Laboratories, UK). The presence of phaeochromocytoma and hyperparathyroidism was excluded by appropriate testing, including measurement of urinary catecholamines, ionized calcium and parathyroid hormone (PTH). The patient was thus submitted to total thyroidectomy. The histological examination revealed an MTC focus of $15 \mathrm{~mm}$ in the right lobe with $1 / 15$ metastatic lymph node (pT1N1b). A papillary microcarcinoma $(5 \mathrm{~mm})$ was also incidentally found in the left lobe. At 3 months after the surgical intervention, both basal and stimulated CT levels were less than $0.2 \mathrm{pg} / \mathrm{ml}$ indicating the remission of the disease.

A complex germline mutation (see below) was found in both the mother and the child. The 4-year-old boy did not show any thyroid nodule at ultrasonography, but his basal CT was $15.8 \mathrm{pg} / \mathrm{ml}$ with pentagastrin-stimulated values of $61.1 \mathrm{pg} / \mathrm{ml}$ at $2 \mathrm{~min}$ after i.v. injection. He was submitted to prophylactic thyroidectomy and the histological examination showed C-cell hyperplasia diffuse in both lobes. At 3 months after the surgical treatment, his basal and stimulated CT levels are less than $0.2 \mathrm{pg} / \mathrm{ml}$ (Fig. 1B).

The proband's father does not show any evidence of thyroid nodule at physical examination or other clinical manifestations of MTC at 76 years of age, and refuses biochemical, instrumental and genetic examinations. All family members are unaware of the existence of relatives with the features reported by Vandenbosch et al. (2005).

\section{Mutation analysis}

An informed consent for DNA analysis was obtained for all screened subjects. DNA was extracted from whole blood by standard methods in the proband, her husband and her child. PCR amplifications of exons 10, 


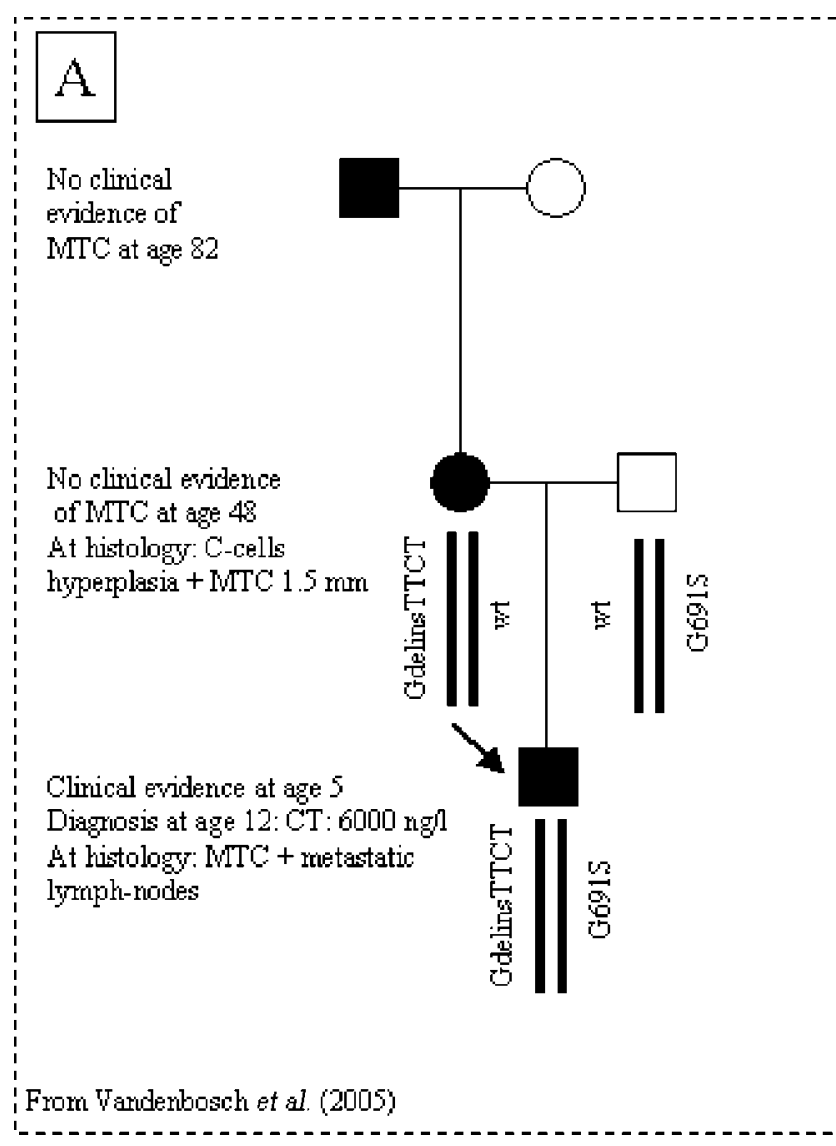

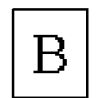

No clirucal evidence of MTC at age 76

Clirical evidence of lMTC at age 41 CT: 225 (basal), 1528 (stirnulated) At histology ITC + metastatic lymph-rodes (pT1N1b)

Clirical eviderce at age 4 CT: 16 (basali/61 (stirnulated) At histolog: bilateral C celle hypenslasia
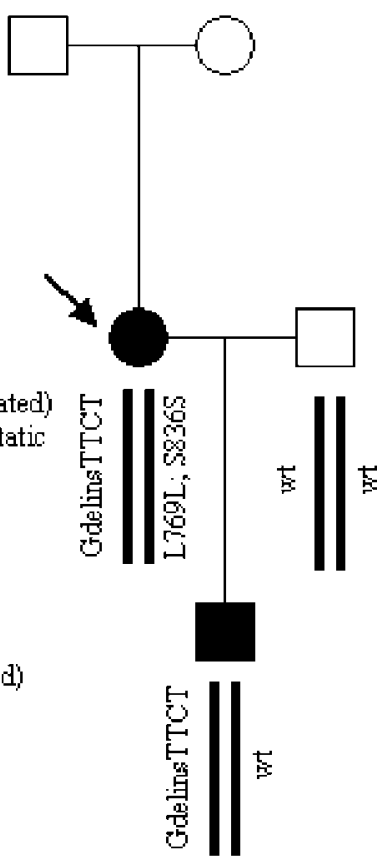

Figure 1 Pedigrees of the family reported by Vandenbosch et al. (2005) (A) and of the present family (B). Members harbouring the mutation are indicated with black symbols. Probands are indicated by arrows. The allele segregation of the mutation (GdelinsTTCT) and the three polymorphisms (G691S, L769L and S836S) are reported for both families.

$11,13,14$ and 15 of the RET gene were performed using primers flanking each exon and PCR products were directly sequenced as previously described (Fugazzola et al. 2002). One sequence read from each direction across the entire coding region and including intron-exon boundaries was obtained. All sequence variants, including polymorphisms, were considered in all studied members. Each DNA extraction, PCR amplification and sequencing was repeated starting from two different blood samples. The experimental studies have been approved by the ethics committees of the Institution.

\section{Functional studies}

\section{Construction of the RET mutant}

Plasmids carrying Ret9-WT (the short isoform of protoRet gene) and Ret9-C634R (the short isoform of protoRet gene containing a MEN2A causing mutation) were kindly donated by Dr Bongarzone and have been described in detail previously (Borrello et al. 1995). Site-directed mutagenesis was performed, on Ret9-WT construct, using an in vitro oligonucleotide mutagenesis system (QuikChange XL site-directed mutagenesis; Stratagene, La Jolla, CA, USA). The mutant clones were identified by amplification refractory mutation system technique using alternatively either a forward primers specific for the wild type (5'-ACCACAAGTTTGCCCACAAGC- $3^{\prime}$ ) or a forward primer specific for the mutant sequence $\left(5^{\prime}\right.$-CACAAGTTTG CCCACAATTCT- $\left.3^{\prime}\right)$, together with a common reverse oligonucleotide (5'-AGGATCTTGAAGGCATCCAC$\left.3^{\prime}\right)$. The full-length cDNA carrying the mutation (delGinsTTCT) was entirely sequenced and the mutant was found to differ from the wild-type Ret only for the mutation. The mutant cDNA was then inserted into a eukaryotic expression vector, pRC-CMV (Invitrogen). Plasmid DNA was extracted using the MAXI PREP kit (Qiagen) as suggested by the supplier.

\section{Cell cultures, transfection and focus formation assay} Transient transfection experiments were performed in human 293 T-cells, $5 \times 10^{5}$, by calcium phosphate 
precipitation, as previously described (Bongarzone et al. 1999). Cells were grown in Dulbecco's modified Eagle's medium (DMEM) containing 10\% calf serum for $72 \mathrm{~h}$.

NIH3T3 cells $\left(2 \times 10^{5}\right)$ were transfected with calcium-phosphate coprecipitation using $1 \mu \mathrm{g}$ plasmid DNA and $25 \mu \mathrm{g}$ mouse carrier DNA. G418-resistant colonies were selected in DMEM $+10 \%$ calf serum supplemented with $500 \mu \mathrm{g} / \mathrm{ml}$ G418 antibiotic and fixed or isolated 14 days after transfection. Transformation foci were selected in DMEM $+5 \%$ calf serum and fixed 21 days after transfection.

The biological effect of the complex mutation was tested in a focus formation assay on NIH3T3 mouse fibroblast cells. The in vitro transforming activity of the RET mutant was tested in comparison with Ret9-wild type (Ret9-WT), while the construct Ret9-C634R was used as a positive control.

\section{Immunoprecipitation and western blot analyses}

Protein samples either from transient or stable transfections were prepared as previously described (Borrello et al. 1995) and immunoprecipitated with a short isoform-specific rabbit polyclonal anti-Ret antiserum (Santa Cruz Biotechnology, Santa Cruz, CA, USA). For dimerization assay, anti-Ret immunoprecipitates were eluted in non-reducing conditions, where 2-mercaptoethanol was excluded from the Laemmli's buffer.

Immunoprecipitates were resolved by electrophoresis on $6 \%$ SDS-PAGE. Proteins were transferred onto nitrocellulose filters, blocked with 5\% BSA and immunoblotted with the monoclonal anti-phosphotyrosine antibody (anti-Ptyr; Upstate Biotechnology, Inc., Lake Placid, NY, USA). The same blot was stripped and reprobed with the anti-Ret antiserum described earlier. Immunoreactive bands were visualized using horseradish peroxidase conjugated anti-rabbit or -mouse antisera and enhanced chemiluminescence detection reagents (Amersham), followed by autoradiography.

\section{Dimerization assay}

Immunoprecipitates from both wild-type and mutant cell lines were subjected to SDS-PAGE under reducing and non-reducing conditions, as previously described (Bongarzone et al. 1999).

\section{Structure prediction and modelling}

The PredictProtein server (http://cubic.bioc.columbia. edu) was used to assess the effect of the delGinsTTCT mutation on the predicted secondary structure of Ret protein (Altschul et al. 1997, Rost et al. 2004). META-PP (http://cubic.bioc.columbia.edu) provides a single-page interface to various World Wide Web services for sequence analysis, homologue retrieval and prediction of protein structure. The algorithms accessed by META-PP include: secondary structure (Jpred, PHDsec, PROFsec, PSIpred, PSSP and Sspro), residue solvent accessibility (PHDacc and PROFacc), transmembrane helix location and topology (PHDhtm, PHDtopology), protein globularity (GLOBE), coiled-coil regions (COILS), Cys bonds (CYSPRED) and structural switching regions (ASP).

\section{Results}

\section{Mutation analysis}

At nucleotide 2646 (exon 11), a $\mathrm{G}$ deletion and a TTCT insertion (c.2646delGinsTTCT) were found in the proband. Both changes are lying on the same allele, since this mutation was transmitted to her son. This in-frame complex variation was absent in a series of 180 control alleles and leads to the substitution of an Asn for a Lys (Lys666Asn) and to a Ser insertion at codon 667 (Fig. 2). The mutated residues are in the intracellular portion of the receptor, immediately after the transmembrane domain. No additional mutations in exons 10, 13, 14 and 15 were detected in the two patients. In the proband, two polymorphisms were found in exon 13 and 14 (Leu769Leu and Ser836Ser), while neither her son nor her husband harbours the above polymorphisms (Fig. 1B).

\section{Functional studies}

To characterize biochemically and biologically the new mutation, we have generated, by site-directed mutagenesis of RET cDNA, the mutant Ret9-delGinsTTCT. The Ret9-delGinsTTCT mutant was entirely sequenced and cloned into the pRC-CMV eukaryotic expression vector carrying the G418 resistance gene. The construct was transiently expressed in $293 \mathrm{~T}$ cells in order to verify the synthesis of the correctly sized protein. The Ret9delGinsTTCT mutant was found to be significantly more phosphorylated than the wild type (data not shown). In order to investigate the biological effect of the mutation, a focus formation assay on NIH3T3 cells was performed. As shown in Fig. 3, Ret9-delGinsTTCT displayed a transforming activity weaker than Ret9C634R, but higher that that of Ret9-WT, indicating that this mutation is able to activate the oncogenic activity of Ret protein in a manner that could justify the delayed onset of the disease compared with MEN2A related mutation. Moreover, the G418 resistant Ret9-delGinsTTCT colonies isolated during the transfection experiment, showed a morphology different from that of the parental NIH3T3 cells and displayed 
a mildly transformed phenotype. To verify if the Ret9delGinsTTCT transforming activity was matched with an increase in the steady state Tyr phosphorylation level, the Ret protein was biochemically analysed in different G418 resistant colonies. As shown in Fig. 4A, Ret9-delGinsTTCT protein is more Tyr phosphorylated than the Ret9-WT protein. Therefore, even in the absence of ligands, Ret9-delGinsTTCT can be phosphorylated, as previously observed for Ret9-C634R. The amount of phosphorylation found for Ret9-delGinsTTCT was however lower than that of Ret9-C634R (Bongarzone et al. 1999), in agreement again with the milder phenotype associated with this complex mutation. Finally, the fully glycosylated form of Ret is more represented in Ret9-delGinsTTCT than in Ret9-WT, indicating that the mutation could stabilize the mature Ret form into the membrane, thus enhancing its transforming potential.

In order to exclude the activation mechanism of the complex mutation, the formation of stable dimers, as reported for Cys mutants in the extracellular domain, anti-Ret immuno-precipitates from cell lines expressing Ret-delGinsTTCT, Ret9-C634R and Ret9-WT were analyzed under non-reducing conditions. As shown in Fig. 4B, the presence of Ret dimers was revealed only in cells expressing Ret9-C634R and not in cells expressing Ret-delGinsTTCT or Ret9-WT proteins.

\section{Possible effects on protein structure}

To assess whether the change of amino acid from Lys to Asn at position 666 followed by the insertion of Ser667 affects the secondary structure or protein folding, we analyzed the whole wild-type and mutant Ret protein sequence by means of several Web-based tools. Most algorithms predicted significant changes to

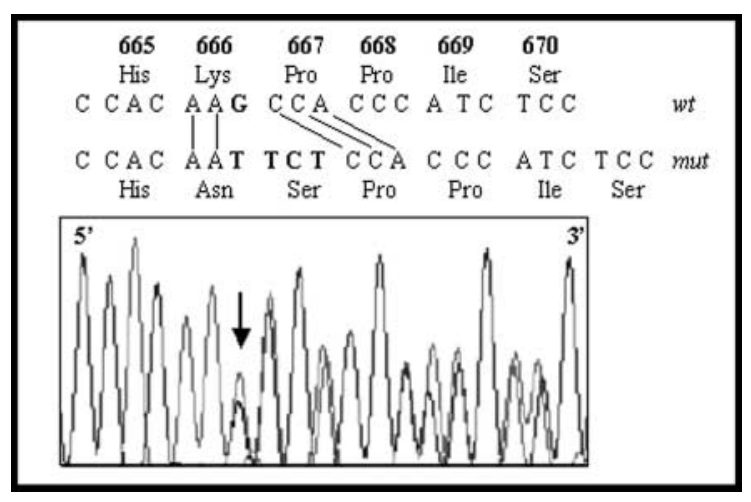

Figure 2 RET genomic DNA forward sequences in the affected members. The complex mutation (c.2646delGinsTTCT) lying in exon 11 is indicated by the arrow. The mutation leads to the substitution of a Lys for an Asn at codon 666 and to a Ser insertion at position 667 . the protein folding as a result of this in-frame mutation (Fig. 5). In particular, the transmembrane $\alpha$-helical acquires a different conformation, which could impair the flexibility of the receptor at that level. Moreover, the content and distribution of $\beta$-strands, in the downstream region close to the insertion, is modified and also the proportion of solvent-exposed residues decreases drastically. Taken together, this mutation is predicted to significantly change the secondary structure of the protein, folding and interaction with a non-aqueous environment.

\section{Discussion}

In the present study, the functional analysis of a complex RET mutation found in a FMTC family is reported. This alteration is of particular interest since it is, overall, the first mutation lying in the juxtamembrane intracellular region of the receptor. The molecular mechanisms eventually leading to Ret constitutive activation include the ligand-independent formation of covalently linked dimers in the case of MEN2A/FMTC mutations involving the Cys-rich domain (Santoro et al. 1995) or modification in the specificities of the substrates that are phosphorylated during intracellular signalling in the case of MEN2B mutations (Songyang et al. 1995). At variance, the molecular mechanism accounting for the gain of Ret function is presently unknown for the mutations lying in other regions of the receptor that were found in FMTC and in sporadic MTC.

The present deletion/insertion cause an in-frame complex mutation located in the intracellular portion of the receptor, nine residues after the transmembrane domain and 55 residues before the beginning of the TK domain. Consistent with the observed phenotype in vivo, a focus formation assay in NIH3T3 cells showed that Ret9-delGinsTTCT displayed a transforming activity weaker than Ret9-C634R, but stronger that that of Ret9-WT. Functional experiments in transient and stably transfected cell lines showed an increased auto-phosphorylation activity of the mutant receptor, indicating that, even in the absence of ligands, Ret9delGinsTTCT can be phosphorylated. Moreover, the mature Ret form was found to be more represented in comparison with Ret9-WT, suggesting that this mutation could stabilize the mature Ret form into the membrane, thus enhancing its transforming potential. As expected, dimerization assay demonstrated that the transforming potential of the Ret9-delGinsTTCT mutation is not mediated by disulphide bond formation. Computational analysis performed by means of the PredictProtein server (Rost et al. 2004) predicted 
NIH

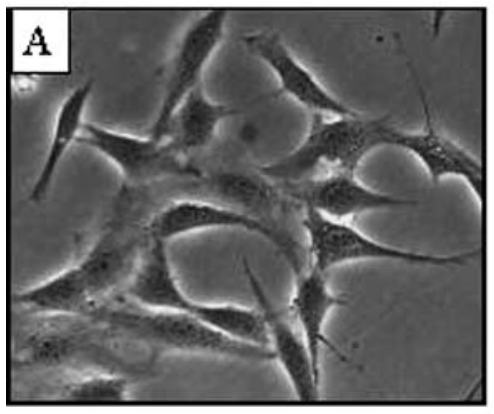

delGinsTTCT

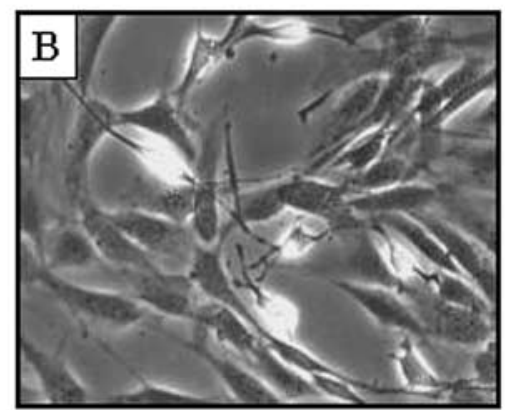

Cys634Arg

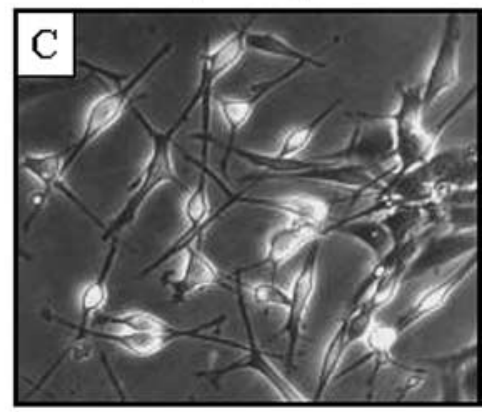

\begin{tabular}{|l|c|}
\hline Constructs (pRC-CMV) & Transformed foci/G-418-resistant colonies \\
\hline Ret9-C634R & $5.8 \times 10^{-2}$ \\
\hline Ret9-delGinsTTCT & $2.1 \times 10^{-3}$ \\
\hline Ret9-WT & $8.5 \times 10^{-4}$ \\
\hline
\end{tabular}

Figure 3 The Ret9-delGinsTTCT complex mutation induces transformation in vitro. (A) Morphology of untransfected NIH3T3 cells; (B) NIH3T3 transfected with the delGinsTTCT mutation displaying the transformed phenotype and (C) NIH3T3 transfected with the MEN2A mutation (Cys634Arg). The transforming activity of Ret9-delGinsTTCT and Ret9-C634R on NIH3T3 is reported in the table.

various modifications induced in the mutant receptor. These include alterations in both transmembrane flexibility and solvent accessibility of the protein. Protein transmembrane flexibility is crucial in ligandbinding kinetics (Teague 2003) and the conformational plasticity is a hallmark of protein kinases (Huse \& Kuriyan 2002). It is therefore likely that this in frame deletion/insertion affects the conformation of the receptor, thus leading to an increased tyrosine kinase domain activity in a ligand-independent manner.

The analysis of allele segregation indicates that the mother has an allele harbouring the RET mutation and another allele harbouring the two polymorphisms in exons 13 and 14. The son inherited the mutated allele

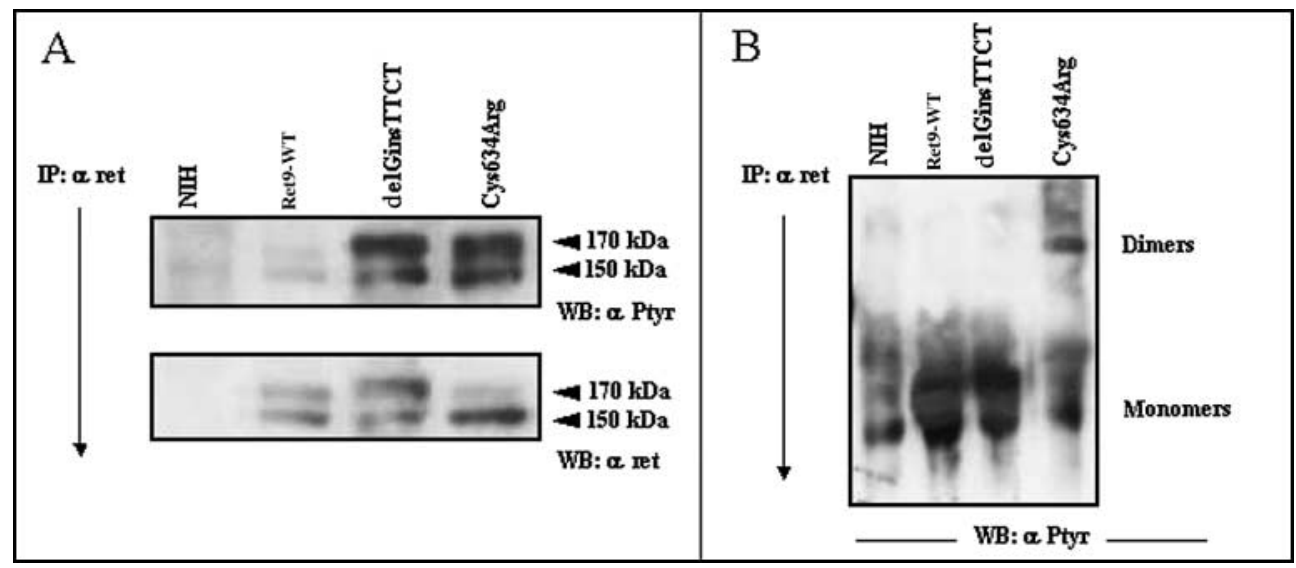

Figure 4 (A) Tyr phosphorylation of Ret receptors. Lysates from NIH3T3 cells transfected with Ret9-WT, Ret9-delGinsTTCT and Ret9-C634R constructs were immunoprecipitated (IP) with anti-Ret antiserum. Western blot filters (WB) were developed with antiphosphotyrosine $(\alpha$-Ptyr) or anti-Ret antiserum $(\alpha$-ret). Ret9-delGinsTTCT protein displays an amount of phosphorylation higher than the Ret9-WT protein and lower than Ret9-C634R. Moreover, the fully glycosylated form of Ret is more represented in Ret9delGins TTCT than in Ret9-WT. (B) Dimerization assay of mutant Ret proteins. Lysates were IP with anti-ret and separated by SDSPAGE (6\%) under non-reducing conditions. WB filters were developed with $\alpha$-Ptyr antiserum. The positions of the Ret dimers and monomers are indicated. The presence of Ret dimers was revealed only in the Ret9-C634R mutant. 

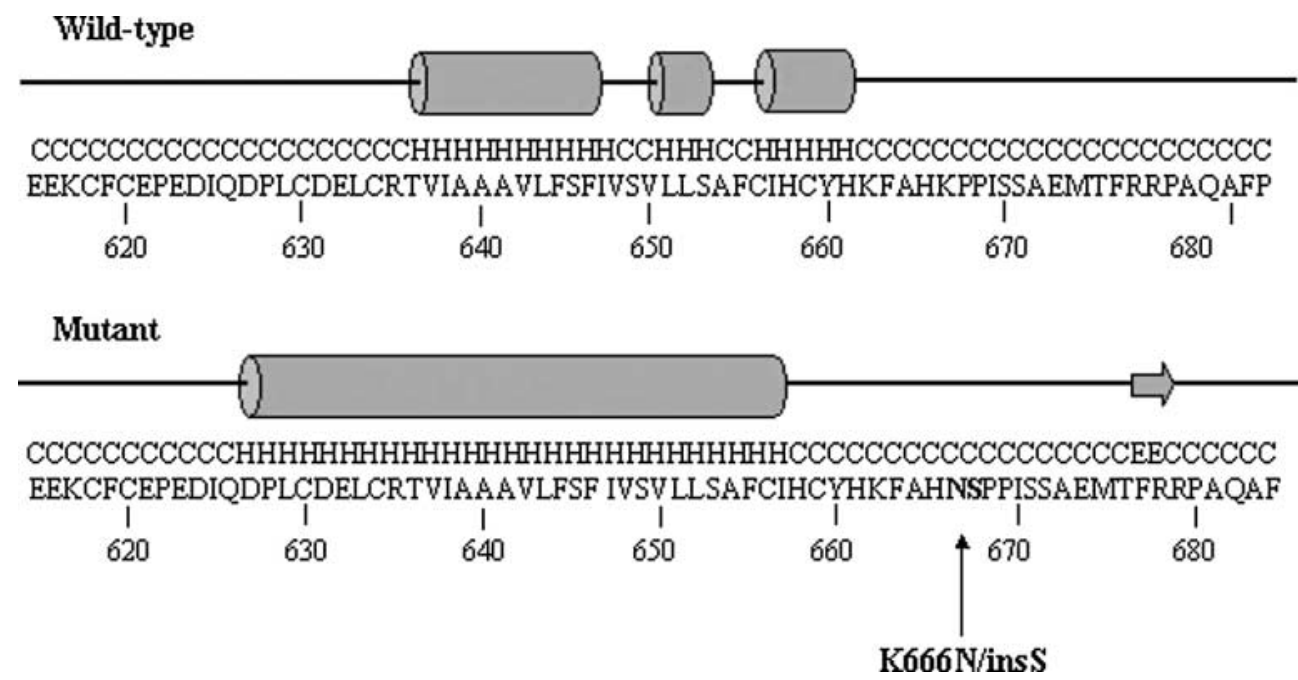

Figure $\mathbf{5}$ The secondary structure of the Ret protein in the vicinity of the reported complex mutation as predicted by PROF (profile network prediction HeiDelberg) and PSI-pred (PSI-BLAST profiles used for prediction). The transmembrane $\alpha$-helix would undergo a conformational change in the mutant protein, which likely reduces the flexibility of the molecule. Moreover, one additional intracellular $\beta$-sheet would be generated in the mutant. $\mathrm{H}$, helix; $\mathrm{E}$, extended (sheet); C, other (loop).

from the mother and a wild-type allele from the father (Fig. 1B). Our data do not confirm the hypothesis of Vandenbosch et al. (2005) suggesting that the G691S polymorphism is necessary for the development of clinical and metastatic MTC and is responsible for the young age of onset. Indeed, such a polymorphism was absent in our family members, despite the presence of metastatic MTC in the mother, and of a C-cells hyperplasia associated with elevated basal and stimulated CT levels in the 4-year-old child. Considering all the clinical data obtained in both families (Vandenbosch et al. 2005 and present paper), this mutation is associated with a phenotype of intermediate severity. Indeed, the child of the first family described had a delayed diagnosis (thyroidectomy was performed 7 years after the first clinical manifestation) and the tumour staging was worse than that observed in the child of the present family. However, the clinical behaviour is compatible with the possible occurrence of 'anticipation' in both families. This phenomenon is characterized by an increase in severity and number of affected members associated with an earlier age of disease onset in succeeding generations. It is commonly found in neuropsychiatric disorders, but also reported in familial cancer syndromes, including familial adenomatous polyposis (FAP) and MEN1 (Presciuttini et al. 1994, Giraud et al. 1997) and has previously been described by us in a multigenerational FMTC family (Fugazzola et al. 2002). Finally, the finding of this novel complex mutation in a total of five apparently unrelated families of Belgian origin argues in favour of a common founder effect. In one of the three families reported in a recent meeting (Bex et al. 2006), a member was found affected with phaeochromocytoma at the age of 48 years. Although this appears to be an isolated case ( $1 / 15$ adult carriers), screening of catecholamine levels is advocated in carriers of this mutation.

In conclusion, we report the functional analysis of the first mutation lying in the juxtamembrane intracellular domain of the receptor indicating that also alterations at this level can activate Ret in a ligand-independent manner. Indeed, functional studies showed increased transforming and auto-phosphorylation activities and higher amounts of fully glycosylated mutant receptor when compared with Ret wild type. Finally, the description of novel mutations, located outside the residues and domains classically involved in MEN2 and FMTC families, indicate that the screening for RET mutations based on restriction analysis may be affected by unexpected false negative results.

\section{Acknowledgements}

The authors are grateful to Dr Italia Bongarzone and Piera Mondellini (Istituto dei Tumori, Milan), for donating plasmids carrying Ret9-WT and Ret9-C634R and for helpful discussion. This study was partially supported by research funds of Istituto Auxologico Italiano IRCCS, Fondazione Policlinico IRCCS and University of Milan, Italy. The authors declare that there is no conflict of interest that would prejudice the impartiality of this scientific work. 


\section{References}

Ahmed SA, Snow-Bailey K, Highsmith WE, Sun W, Fenwick RG \& Mao R 2005 Nine novel germline gene variants in the RET proto-oncogene identified in twelve unrelated cases. Journal of Molecular Diagnostics 7 283-288.

Alberti L, Borrello MG, Ghizzoni S, Torriti F, Rizzetti MG \& Pierotti MA 1998 Grb2 binding to the different isoforms of Ret tyrosine kinase. Oncogene 17 1079-1087.

Alemi M, Lucas SD, Sallstrom JF, Bergholm U, Akerstrom G \& Wilander E 1997 A complex nine base pair deletion in RET exon 11 common in sporadic medullary thyroid carcinoma. Oncogene 14 2041-2045.

Altschul S, Madden T, Shaffer A, Zhang J, Zhang Z, Miller W \& Lipman D 1997 Gapped Blast and PSIBlast: a new generation of protein database search programs. Nucleic Acids Research 25 3389-3402.

Bex M, Decallonne B, Matthijs G \& Legius E 2006 The rare RET mutation (insTTCTdelG) at codon 666 is associated with a low penetrance of medullary thyroid carcinoma and pheochromocytoma. 8th European Congress of Endocrinology, 1-5 April 2006, Glasgow (UK), P501.

Bongarzone I, Vigano' E, Alberti L, Mondellini P, Uggeri M, Pasini B, Borrello MG \& Pierotti M 1999 The Glu632Leu633 deletion in cysteine rich domain of Ret induces costitutive dimerization and alters the processing of the receptor protein. Oncogene 18 4833-4838.

Borrello MG, Smith DP, Pasini B, Bongarzone I, Greco A, Lorenzo MJ, Arighi E, Miranda C, Eng C, Alberti L et al. 1995 RET activation by germline MEN 2A and MEN 2B mutations. Oncogene 11 2419-2427.

Ceccherini I, Pasini B, Pacini F, Gullo M, Bongarzone I, Romei C, Santamaria G, Matera I, Mondellini P, Scopsi L et al. 1997 Somatic in frame deletions not involving juxtamembranous cysteine residues strongly activate the RET proto-oncogene. Oncogene 14 2609-2612.

Eng C \& Mulligan LM 1997 Mutations of the RET protooncogene in the multiple endocrine neoplasia type 2 syndromes, related sporadic tumors and Hirschsprung disease. Human Mutation 9 97-109.

Fugazzola L, Cerutti N, Mannavola D, Ghilardi G, Alberti L, Romoli R \& Beck-Peccoz P 2002 Multigenerational familial medullary thyroid cancer (FMTC): evidence for FMTC phenocopies and association with papillary thyroid cancer. Clinical Endocrinology 56 53-63.

Giraud S, Choplin H, Teh BT, Lespinasse J, Jouvet A, Labat-Moleur F, Lenoir G, Hamon B, Hamon P \& Calender A 1997 A large multiple endocrine neoplasia type 1 family with clinical expression suggestive of anticipation. Journal of Clinical Endocrinology and Metabolism 10 3487-3492.

Hansford JR \& Mulligan LM 2000 Multiple endocrine neoplasia type 2 and RET: from neoplasia to neurogenesis. Journal of Medical Genetics 37 817-827.

Hofstra RMW, Stelwagen T, Stulp RP, De Long D, Hulsbeek M, Kamsteeg EJ, Van Den Berg A, Landsvater RM, Vermey A, Molenaar WM et al. 1996 Extensive mutation scanning of
RET in sporadic medullary thyroid carcinoma and of RET and VHL in sporadic pheocromocytoma reveals involvement of these genes in only a minority of cases. Journal of Clinical Endocrinology and Metabolism 81 2881-2884.

Hoppner W \& Ritter MM 1997 A duplication of 12 bp in the critical cysteine rich domain of the RET proto-oncogene results in a distinct phenotype of multiple endocrine neoplasia type 2A. Human Molecular Genetics 6 587-590.

Hoppner W, Dralle H \& Brabant G 1998 Duplication of 9 base pairs in the critical cysteine-rich domain of the RET proto-oncogene causes multiple endocrine neoplasia type 2A. Human Mutation (Suppl 1) S128-S130.

Huse M \& Kuriyan J 2002 The conformational plasticity of protein kinases. Cell 109 275-282.

Kalinin V \& Frilling A 1998 27-bp deletion in the ret protooncogene as a somatic mutation associated with medullary thyroid carcinoma. Journal of Molecular Medicine 76 365-367.

Kimura T, Yoshimoto K, Yokogoshi Y \& Saito S 1995 Mutations in the cysteine-rich region of the RET proto-oncogene in patients diagnosed as having sporadic medullary thyroid carcinoma. Endocrine Journal 42 517-525.

Marsh DJ, Andrew SD, Learoyd DL, Pojer R, Eng C \& Robinson BG 1998 Deletion-insertion mutation encompassing RET codon 634 is associated with medullary thyroid carcinoma. Human Mutation (Suppl 1) S3-S4.

Pigny P, Bauters C, Wemeau JL, Lecomte Houcke M, Crepin M, Caron P, Giraud S, Calender A, Buisine MP, Kerckaert JP et al. 1999 A novel 9-base pair duplication in RET exon 8 in familial medullary thyroid carcinoma. Journal of Clinical Endocrinology and Metabolism 84 1700-1704.

Presciuttini S, Varesco L, Sala P, Gismondi V, Rossetti C, Bafico A, Ferrara C \& Bertario L 1994 Age of onset in familial adenomatous polyposis: heterogeneity within families and among APC mutations. Annals of Human Genetics 58 331-342.

Quadro L, Fattoruso O, Cosma MP, Verga U, Porcellini A, Libraia A \& Colantuoni V 2001 Loss of heterozygosity at the RET protoncogene locus in a case of multiple endocrine neoplasia type 2A. Journal of Clinical Endocrinology and Metabolism 86 239-244.

Romeo G, Ronchetto P, Luo Y, Barone V, Seri M, Ceccherini I, Pasini B, Bocciardi R, Lerone M, Kaariainen H et al. 1994 Point mutations affecting the tyrosine kinase domain of the RET proto-oncogene in Hirschsprung's disease. Nature 367 377-378.

Rost B, Yachdav G \& Liu J 2004 The PredictProtein server. Nucleic Acids Research 32 W321-W326.

Santoro M, Carlomagno F, Romano A, Bottaro DP, Dathan NA, Greco M, Fusco A, Vecchio G, Matoskova B, Kraus MH et al. 1995 Activation of RET as dominant transforming gene by germline mutations in MEN 2A and MEN 2B. Science 267 381-383.

Songyang Z, Carraway KL, Eck MJ, Harrsion SC, Feldman RA, Mohammadi M, Schlessinger J, Hubbard SR, Smith DP, 
Eng C et al. 1995 Catalytic specificity of protein-tyrosine kinases is critical for selective signalling. Nature $\mathbf{3 7 3}$ 536-539.

Teague SJ 2003 Implications of protein flexibility for drug discovery. Nature Reviews Drug Discovery 2 527-541. Uchino S, Noguchi S, Yamashita H, Sato M, Adachi M, Yamashita H, Watanabe S, Ohshima A, Mitsuyama S, Iwashita T et al. 1999 Somatic mutations in RET exons 12 and 15 in sporadic medullary thyroid carcinomas: different spectrum of mutations in sporadic type from hereditary type. Japanese Journal of Cancer Research 90 1231-1237.

Vandenbosch B, Renard M, Uyttebroeck S, Sciot R, Matthijs G \& Legius E 2005 Medullary thyroid carcinoma in a child with a new RET mutation and a RET polymorphism. Genetic Counseling 16 95-100. 